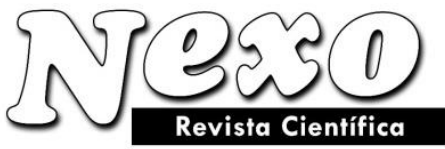

ISSN-E 1995-9516

Universidad Nacional de Ingeniería COPYRIGHT (C) (UNI). TODOS LOS DERECHOS RESERVADOS http://revistas.uni.edu.ni/index.php/Nexo https://doi.org/10.5377/nexo.v33i02.10809

\title{
Características de la actividad sísmica de Cuba
}

\section{Characteristics of seismic activity in Cuba}

\author{
Mario Octvio Cotilla Rodríguez \\ Universidad Complutense de Madrid. Facultad de Ciencias Físicas. Departamento de Física de la Tierra y \\ Astrofísica. Ciudad Universitaria s/n. 28040, Madrid \\ Correo: macot@ucm.es
}

(recibido/received: 02-May-2020; aceptado/accepted: 25-September-2020)

\begin{abstract}
RESUMEN
Se presenta un análisis sobre la actividad sísmica de Cuba. Esta isla tiene una $\mathbf{M}_{\text {máx }}$ de 7,7 que está asociada a las inmediaciones de Cabo Cruz, con la falla Oriente. El mayor peligro sísmico está en la ciudad de Santiago de Cuba. La sismicidad de las islas vecinas ha afectado a Cuba, y ellas tienen un mayor nivel de actividad, incluida la tsunamigénica. Se da un diagnóstico de terremotos fuertes.
\end{abstract}

Palabras claves: Actividad sísmica, Cuba, sismicidad, terremoto.

\begin{abstract}
An analysis of the Cuba`s seismic activity is presented. This island has a $\mathbf{M}_{\max }$ of 7.7 that is associated to the vicinity of Cabo Cruz, with the Oriente fault. The greatest seismic hazard is in the city of Santiago de Cuba. The seismicity of neighboring islands has affected Cuba, and they have a higher level of activity, including the tsunami genetic. A diagnosis of strong earthquakes is given.
\end{abstract}

Keywords: Cuba, earthquake, seismicity, seismic activity.

\section{INTRODUCC|IÓN}

El asentamiento inicial de los europeos en el siglo XV se situó, mayoritariamente, en la región septentrional del Caribe (Cuba, Jamaica, La Española y Puerto Rico (Figura 1)). Así aparecen los primeros reportes sobre terremotos y tsunamis en América. Es importante indicar que los colonizadores: 1) conocían sobre esos fenómenos naturales en Europa; 2) que redactaban los informes sobre esos eventos tenían una adecuada preparación intelectual, por lo que no había defecto de forma en los escritos. De esos documentos se deriva a los catálogos de terremotos.

La Tabla 1 contiene la información básica de las 3 regiones físico-geográficas de Cuba relacionadas con los terremotos perceptibles. De ellas es factible valorar el año de fundación de las ciudades y la cantidad de habitantes, que pueden ser afectados por esos eventos. 
Los objetivos principales del trabajo, enmarcados en el Proyecto Científico KUK-AHPAN RTI2018094827-B-C21, son dos: 1) mostrar, sintéticamente, que la actividad sísmica [AS] es diferencial en el Caribe Septentrional donde se localiza Cuba, nuestra área de interés; 2) que esa AS es importante en Cuba, aunque de menor nivel que en los Países vecinos.

Tabla 1. Datos de 28 localidades de 3 regiones de Cuba relacionadas con la perceptibilidad de terremotos.

\begin{tabular}{ccccc}
\hline$-1-$ & $-2-$ & $-1-$ & $-2-$ & $-1-$ \\
\hline Artemisa/ 1818/ 59,13 & Occ & Caibarién/ 1832/ 38,479 & C-Oc & Banes/ 1513/ 34,452 \\
Candelaria/ 1809/ 19,523 & & Camagüey/ 1528/ 769,863 & Or \\
Guane/ 1878/ 35,718 & & Cienfuegos/ 1819/ 164,924 & Baracoa/ 1511/ 79,797 \\
Jagüey Grande/ 1840/87,771 & & Gibara/ 1720/ 71,126 & Cabomo/ 1513/ 235,107 Cruz/ 1494/ 0,1 \\
La Habana/ 1514/ 2.141,652 & Holguín/ 1545/ 350,191 & Guantánamo/ 1796/ 228,436 \\
Matanzas/ 1693/ 145,246 & Remedios/ 1513/ 45,621 & Manzanillo/ 1792/ 150,199 \\
Pinar del Río/ 1867/ 188,614 & Sancti Spíritus/ 1514/ & Moa/ 1939/ 57,652 \\
San Cristóbal/ 1830/ 29,119 & Santa Clara/ 1689/ 784,244 & Pilón/ 1779/ 29,456 \\
San José Lajas/ 1778/ 73,136 & Trinidad/ 1513/ 51,994 & Sagua de Tánamo/ 1735/ 46,967 \\
\hline & & & Santiago de Cuba/ 1515/ 444,851
\end{tabular}

\section{TECTÓNICA Y SISMICIDAD}

La preparación del epígrafe se fundamentó, principalmente, en los siguientes trabajos (Álvarez et al., 1990, 1999; Cotilla-Rodríguez, 1993; Cotilla, 2007, 2010, 2011, 2012; Cotilla y Córdoba, 2011, 2018; Cotilla y Udías, 1999; Cotilla et al., 1988; González et al., 2003; Mann y Burke, 1984; Rosencratz y Mann, 1991; Wiggins y Atakan, 2005). De esta forma se sostiene que Cuba es un archipiélago de las Antillas Mayores, y desde el punto de vista neotectónico un megabloque emergido, y en ascenso diferencial entre sus partes, que está incorporado tectónicamente al borde meridional de la placa Norteamérica (Figuras 1 y 2). Esta neo-estructura tiene 3 grandes bloques (Occidental, Centro-Occidental y Oriental) con 5 límites tectónicos definidos (fallas: Cauto-Nipe, Cochinos, Nortecubana, Oriente y Surcubana) (Figura 3). La complejidad de las estructuras geológica y tectónica de Cuba se evidencian en: 1) la deformación en arco cóncavo al sur en la inmediaciones de Matanzas; 2) los cortes geológicos y geofísicos en que aparecen apiladas, mezcladas y dislocadas diferentemente secuencias rocosas de distintas zonas estructuro-formacionales. 


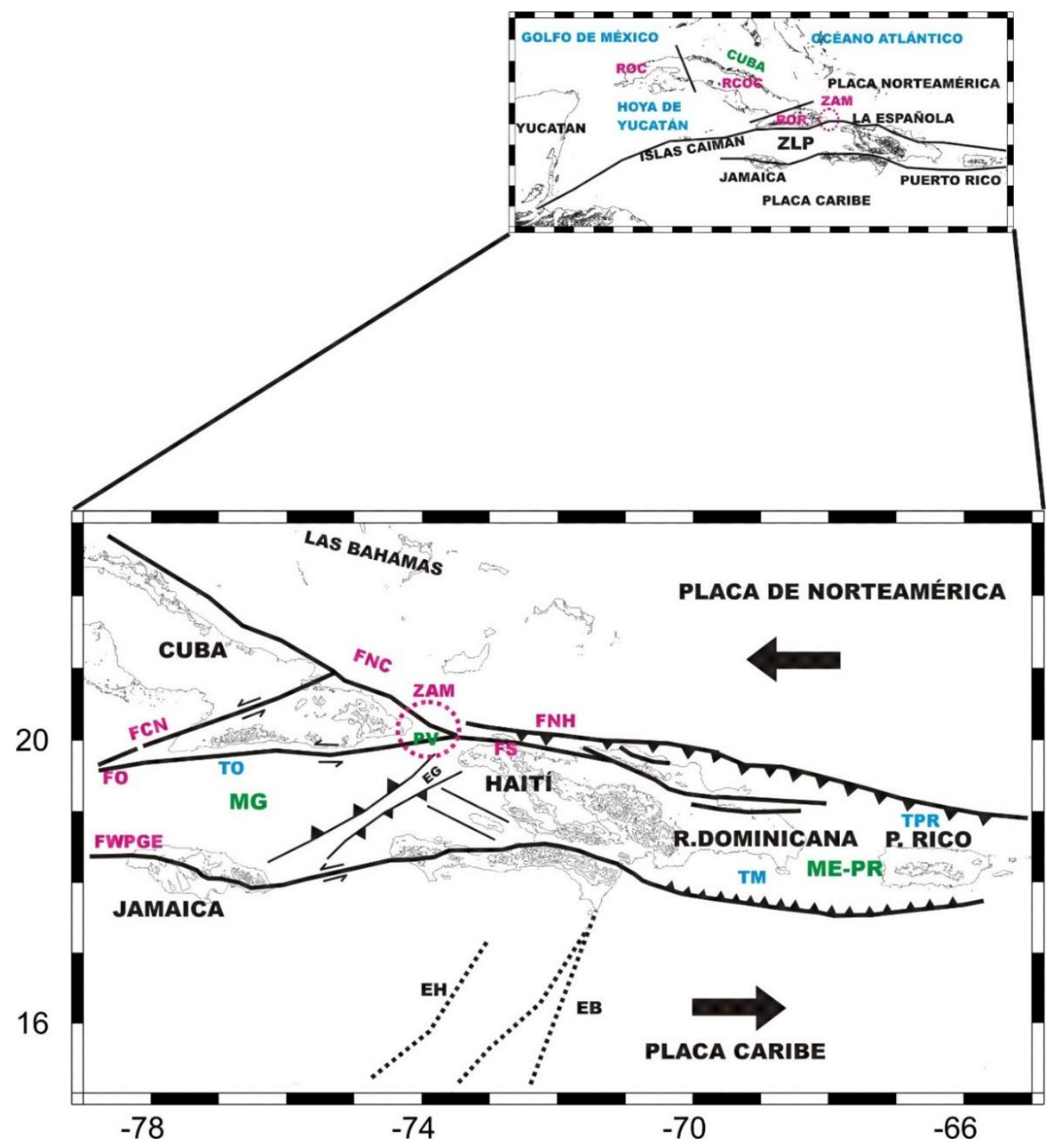

Figura 1. Localización de Cuba.

Aparecen: 1) ZLP=Zona Límite de Placas; ) regiones con letras rojas (ROC=Occidental, RCOC=Centro Occidental, $\mathrm{ROR}=$ Oriental); $\underline{\mathbf{3}}$ ) fallas (líneas gruesas negras con letras rojas: $\mathrm{FCH}=$ Cauto límite, $\mathrm{FNC}=$ Nortecubana, $\mathrm{FO}=$ Oriente, $\mathrm{FS}=$ Septentrional, FSC=Surcubana; FWPGE=Swan-Walton-Plantain Garden-Enriquillo); 4) trincheras oceánicas en letra azul (TM=Muertos; TO=Oriente; TPR=Puerto Rico); $\underline{\mathbf{5}}$ ) sentido de movimiento de las placas (flechas negras gruesas; $\underline{\text { 6) }}$ microplacas con letras verdes (MG=Gonave, MH-PR=Española-Puerto Rico); 7) Elevado (EB=Beata; EG=Gonave, EH=Hess); 8) ZAM=Zona de articulación Maisí (letras en rojo).

Las islas vecinas de Jamaica y La Española (Haití y República Dominicana), en relación con Cuba, tienen una mayor actividad neotectónica y complejidad geodinámica; por lo que su AS es superior (Tablas 2 y 3 ). Ellas están localizadas en la placa Caribe, donde destacan en el plano neotectónico la diferente intensidad en la tendencia: 1) de giro; 2) al levantamiento, de los bloques delimitados. La Tabla 2 muestra el incremento de la perceptibilidad de los terremotos en el tiempo. Esto se explica, lógicamente, a partir del poblamiento sistemático de los territorios. Destaca en la Tabla 3 que: 1) La Española y Jamaica tienen la 
mayor cantidad de tsunamis locales, 13 y 8, respectivamente; 2) Haití, es la zona más activa; $\underline{\text { 3}}$ ) Cuba sólo tiene 2 tsunamis locales, y es la de menor peligro.

Tabla 2. Resumen de las cantidades, aproximadas, de terremotos significativos y fallecidos.

\begin{tabular}{ccccccccc}
\cline { 2 - 7 } & \multicolumn{7}{c}{ Terremotos (Siglos) } & \\
\hline País & XVI & XVII & XVIII & XIX XX & XXI & Total & Fallecidos \\
\hline Cuba & 1 & 1 & 1 & 7 & 9 & 4 & 23 & $<100$ \\
Guatemala & 1 & 0 & 4 & 2 & 14 & 7 & 28 & $>25.000$ \\
Haití & 2 & 5 & 9 & 13 & 12 & 13 & 54 & 350.000 \\
Total & 4 & 6 & 14 & 22 & 35 & 24 & 105 & $>375.000$ \\
\hline
\end{tabular}

Tabla 3. Tsunamis del Caribe Septentrional.

\begin{tabular}{|c|c|c|}
\hline País & Año/ Total & \\
\hline Haití & 1751 (dos); 1755; 1769; 1770; 1775 (dos); 1842; 1860; 1887/ 10 & \\
\hline República Dominicana & $1751 ; 1946 ; 1953 / 3$ & La Española= 13 \\
\hline Jamaica & $1688 ; 1692 ; 1780 ; 1781 ; 1787 ; 1812 ; 1881 ; 1907 / 8$ & \\
\hline Puerto Rico & $1918(\mathrm{dos}) ; 1946 ; 1989 / 4$ & \\
\hline Islas Vírgenes & $1690 ; 1867 ; 1874 / 3$ & \\
\hline Cuba & $\begin{array}{l}1755 \text { (Lisboa); } 1867 \text { (I. Vírgenes); } 1931 \text { y } 1939 \text { (falla } \\
\text { Nortecubana)/ } 4\end{array}$ & \\
\hline
\end{tabular}

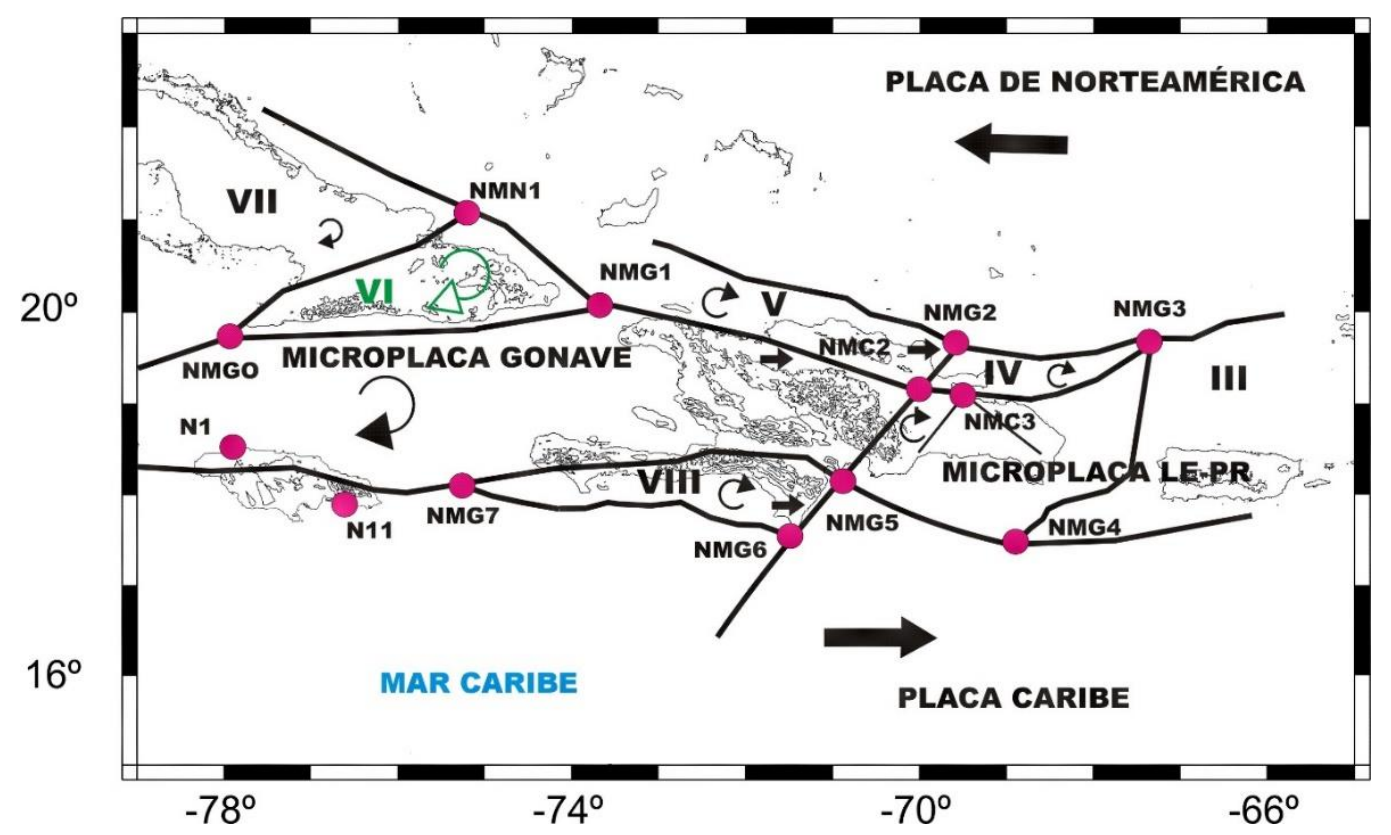

Figura 2. Geodinámica del Este de Cuba

Aparecen: $\underline{\mathbf{1}}$ ) bloques (números romanos); $\underline{\mathbf{2}}$ ) nudos de articulación en color rojo (N1); $\underline{\mathbf{3}}$ ) sentido de movimiento de las placas (flecha negra gruesa); $\mathbf{4}$ ) fallas líneas negras continuas; $\underline{\mathbf{5}}$ ) sentido de desplazamiento de bloques (flechas negras pequeñas); $\underline{\mathbf{6}}$ movimiento de giro de los bloques y microplacas. 
La AS de Cuba se resume en las Tablas 4 y 5. Ella contiene 23 terremotos, considerados por el autor como los principales. Aparecen 15 características de esos eventos sísmicos. Esto permite sostener que todo el territorio es sismo-activo; pero la región suroriental tiene el mayor nivel ( $\mathbf{M}_{\text {máx }}$, cantidad de eventos y frecuencia de repetición). La Figura 4 es una regionalización de la sismicidad en 4 niveles de Intensidad Sísmica (MSK), de 5 a 8 grados, para T=100 años. Otro elemento del análisis está en la Tabla 6. Aparecen 15 terremotos de los territorios más cercanos que han afectado a Cuba, en particular a su parte Oriental.

Tabla 4. Datos de los principales terremotos de Cuba.

\begin{tabular}{|c|c|c|c|c|c|c|c|c|}
\hline $\mathrm{N}^{\circ}$ & $-1-$ & $-2-$ & $-3-$ & $-4-$ & $-5-$ & $-6-$ & $-7-$ & $-8-$ \\
\hline 1 & $1852.07 .07 / 12: 25$ & $7,7 / 5$ & $19,778,4 / 30$ & & & Oriente & $>1.200 / \mathrm{I}$ & Suroriental \\
\hline 2 & 2020.01.28/19:10:24 & $7,7 / 3$ & $19,41978,156 / 14,9$ & & & & & \\
\hline 3 & 1766.06.11/ 00:00 & $6,8 / 9$ & $19,976,1 / 30$ & 80 & $40 / \sim 700$ & & & \\
\hline 4 & 1842.07.07/ & $6,8 / 8$ & $19,7575,35 / 30$ & 75 & 2/ 10 & & & \\
\hline 5 & $1992.05 .25 / 16: 55: 04$ & $6,8 / 7$ & $19,6177,87 / 23$ & 65 & $/ 40$ & & & \\
\hline 6 & 1678.02.11/ 14:59 & $6,75 / 8$ & $19,976,0 / 30$ & 55 & $1 / 3$ & & & \\
\hline 7 & $1932.02 .03 / 06: 15: 03$ & $6,75 / 8$ & $19,6075,313 / 25$ & 25 & $25 / 350$ & & & \\
\hline 8 & $1947.08 .07 / 00: 40: 27$ & $6,6 / 7$ & $19,60075,313 / 35$ & 20 & 1/ varios & & & \\
\hline 9 & 1551.10.18/ 12:00 & $6,6 / 8$ & $19,677,8 / 15$ & 50 & 17 & & & \\
\hline 10 & 1858.01.28/22:04 & $6,5 / 7$ & $19,976,0 / 30$ & & $1 / 2$ & & & \\
\hline 11 & 1800.10.14/ & $6,4 / 8$ & $19,975,9 /$ & 50 & $5 / 30$ & & & \\
\hline 12 & 1852.08.20/ 14:05 & $6,4 / 9$ & $19,7575,32 / 30$ & 75 & $2 / \sim 200$ & & & \\
\hline 13 & 1826.09.18/09:29:00 & $6,4 / 9$ & $19,7575,35 / 30$ & 55 & $3 / 20$ & & & \\
\hline 14 & 2007.02.04/20:56:59 & $6,2 / 4$ & $19,37278,512 / 10$ & 40 & & & & \\
\hline 15 & 1976.02.19/13:59:59 & $5,7 / 8$ & $19,8776,87 / 15$ & 20 & $1 / 8$ & & & \\
\hline 16 & 1914.02.28/ 05:19 & $6,2 / 8$ & $21,3076,20 / 50$ & 15 & & Nortecubana & $1.100 / \mathrm{II}$ & $\begin{array}{l}\text { Centro- } \\
\text { Occidental }\end{array}$ \\
\hline 17 & 1939.08.15/03:52:35 & $5,6 / 7$ & $22,5079,25 /$ & 20 & & & & y Oriental \\
\hline 18 & 1998.12.28/ & $5,6 /$ & 20,78 74,67/ 10 & & & & & \\
\hline 19 & 2014.01.09/05:25:29 & $5,1 / 6$ & $23,1980,68 / 10$ & & & & & \\
\hline 20 & 1880.01.23/04:30:00 & $6,2 / 8$ & $22,783,0 /<20$ & 35 & $3 / \sim 20$ & Guane & 280/ III & Occidental \\
\hline 21 & 1982.12.16/20:20 & $5,0 / 6$ & $22,6181,23 / 30$ & 5 & / 2 & $\begin{array}{l}\text { Cochinos- } \\
\text { Habana- } \\
\text { Cienfuegos }\end{array}$ & $120-300 /$ IV & \\
\hline 22 & 1995.03.09/18:29:13 & $2,1 / 5$ & $22,9082,21 / 3$ & & & & & \\
\hline 23 & 2015.01.21/04:07:10 & $4,1 / 5$ & $22,21681,422 / 16$ & & & & & \\
\hline & Total $=23$ eventos & M2,1-7,7 & $7 \quad$ Tot & ales $=$ & $90 / \sim 1.400 /$ & & & \\
\hline
\end{tabular}

\section{SISMOTECTÓNICA}

El epígrafe se sustenta en los siguientes trabajos (Cotilla-Rodríguez, 1993; Cotilla y Córdoba, 2018; Cotilla y Udías, 1999; Cotilla et al., 1988). De ellos se conoce que Cuba posee un mapa sismotectónico y que éste se ha mejorado sucesivamente en el tiempo (Figura 5). En él se considera que Cuba es una Provincia Sismotectónica activa, en la parte meridional de la placa de Norteamérica, con 4 Unidades, siendo la Suroriental la más importante (Tablas 7 y 8). Sin embargo, su AS es inferior con respecto a las regiones vecinas (Tabla 9). 
Tabla 5. Datos complementarios de la Tabla 4.

\begin{tabular}{ccccccccccccccccc}
\hline $\mathrm{N}^{\circ}$ & $-9-$ & $-10-$ & $-11-$ & $-12-$ & $-13-$ & $-14-$ & $-15-$ & $\mathrm{N}^{\circ}$ & $-9-$ & $-10-$ & $-11-$ & $-12-$ & $-13-$ & $-14-$ & $-15-$ \\
\hline 1 & $\mathrm{X}$ & & $\mathrm{X}$ & $\mathrm{X}$ & $\mathrm{X}$ & & & 13 & & 50 & $\mathrm{X}$ & $\mathrm{X}$ & $\mathrm{X}$ & $\mathrm{X}$ & \\
2 & $\mathrm{X}$ & & $\mathrm{X}$ & & $\mathrm{X}$ & & $\mathrm{X}$ & 14 & $\mathrm{X}$ & 50 & $\mathrm{X}$ & & $\mathrm{X}$ & & $\mathrm{X}$ \\
3 & $\mathrm{X}$ & 85 & $\mathrm{X}$ & $\mathrm{X}$ & & $\mathrm{X}$ & & 15 & $\mathrm{X}$ & 55 & $\mathrm{X}$ & & $\mathrm{X}$ & $\mathrm{X}$ & $\mathrm{X}$ \\
4 & & 77 & $\mathrm{X}$ & $\mathrm{X}$ & $\mathrm{X}$ & $\mathrm{X}$ & & 16 & $\mathrm{X}$ & 25 & $\mathrm{X}$ & & $\mathrm{X}$ & $\mathrm{X}$ & \\
5 & $\mathrm{X}$ & 65 & $\mathrm{X}$ & & $\mathrm{X}$ & $\mathrm{X}$ & $\mathrm{X}$ & 17 & $\mathrm{X}$ & 19 & $\mathrm{X}$ & & $\mathrm{X}$ & $\mathrm{X}$ & $\mathrm{X}$ \\
6 & & 55 & $\mathrm{X}$ & $\mathrm{X}$ & & & & 18 & $\mathrm{X}$ & 9 & & & $\mathrm{X}$ & $\mathrm{X}$ & $\mathrm{X}$ \\
7 & $\mathrm{X}$ & 65 & $\mathrm{X}$ & & $\mathrm{X}$ & $\mathrm{X}$ & $\mathrm{X}$ & 19 & $\mathrm{X}$ & 9 & & & $\mathrm{X}$ & $\mathrm{X}$ & $\mathrm{X}$ \\
8 & $\mathrm{X}$ & 49 & $\mathrm{X}$ & & $\mathrm{X}$ & $\mathrm{X}$ & $\mathrm{X}$ & 20 & $\mathrm{X}$ & 40 & $\mathrm{X}$ & $\mathrm{X}$ & $\mathrm{X}$ & $\mathrm{X}$ & \\
9 & $\mathrm{X}$ & 40 & $\mathrm{X}$ & $\mathrm{X}$ & $\mathrm{X}$ & $\mathrm{X}$ & & 21 & $\mathrm{X}$ & 34 & $\mathrm{X}$ & & $\mathrm{X}$ & $\mathrm{X}$ & $\mathrm{X}$ \\
10 & & 40 & $\mathrm{X}$ & $\mathrm{X}$ & $\mathrm{X}$ & $\mathrm{X}$ & & 22 & $\mathrm{X}$ & 10 & $\mathrm{X}$ & & $\mathrm{X}$ & $\mathrm{X}$ & $\mathrm{X}$ \\
11 & & 40 & $\mathrm{X}$ & $\mathrm{X}$ & $\mathrm{X}$ & $\mathrm{X}$ & & 23 & $\mathrm{X}$ & 10 & $\mathrm{X}$ & & $\mathrm{X}$ & $\mathrm{X}$ & $\mathrm{X}$ \\
12 & $\mathrm{X}$ & 75 & $\mathrm{X}$ & $\mathrm{X}$ & $\mathrm{X}$ & $\mathrm{X}$ & & & & & & & & &
\end{tabular}

Tabla 6. Algunos terremotos de otros Países que afectaron Cuba.

\begin{tabular}{ccccccccc}
\hline Fecha & M & País & Fecha & M & País & Fecha & M & País \\
\hline 1842.07 .05 & 8,2 & Haití & 1943.07 .29 & 7,7 & R. Dominicana & 1692.06 .07 & 7,5 & Jamaica \\
1887.09 .23 & 7,9 & & 1946.08 .04 & 7,5 & & 1899.06 .14 & 7,3 & \\
1770.06 .04 & 7,5 & & 1670.05 .02 & 8,0 & Puerto Rico & 1971.04 .01 & 7,1 & \\
2010.01 .12 & 7,0 & & 1787.05 .02 & & & 1907.01 .14 & 6,6 & \\
2004.11 .21 & 7,0 & & 1918.10 .11 & 7,5 & & 1914.08 .03 & 6,0 & \\
\hline
\end{tabular}

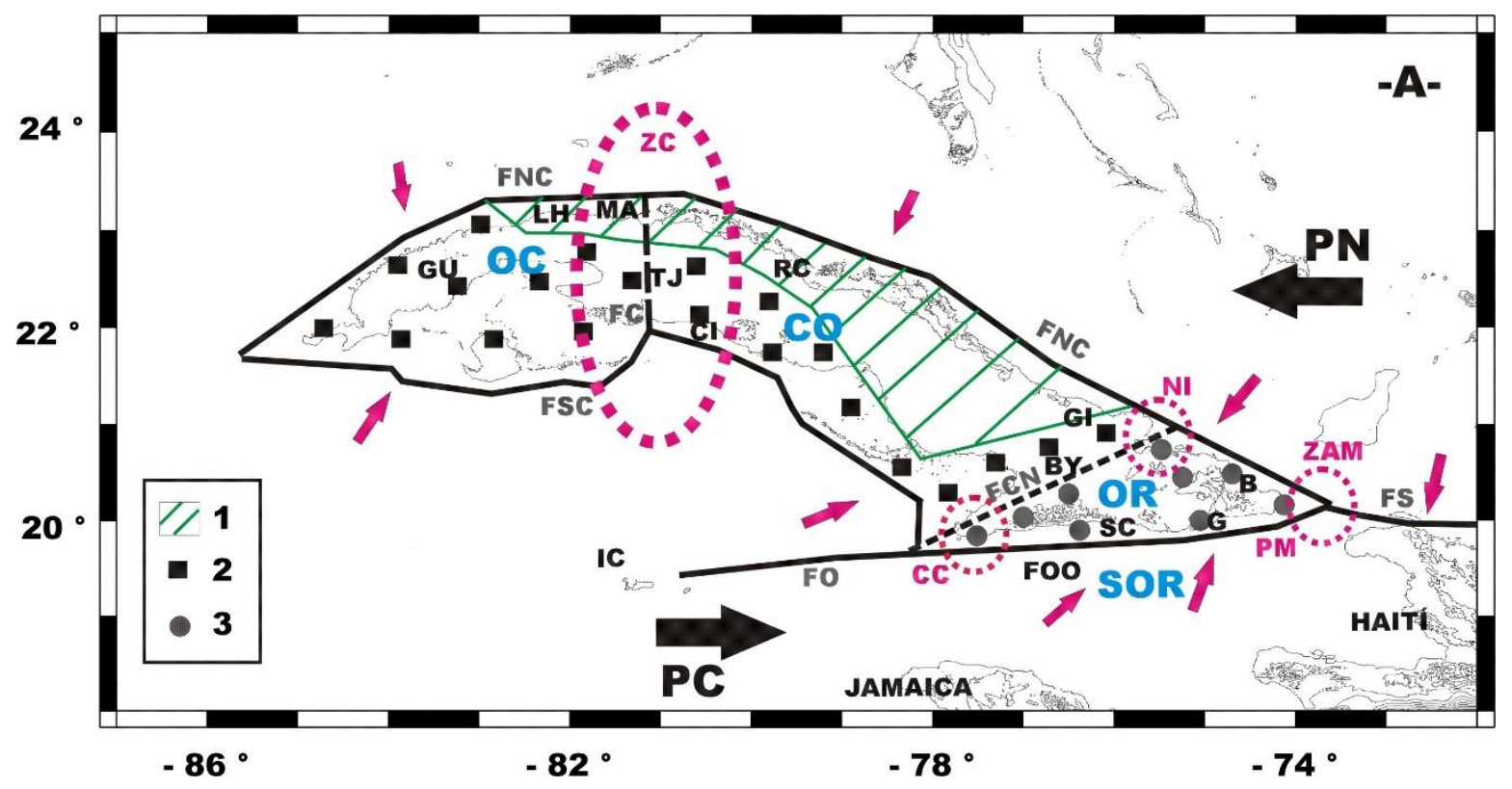

Figura 3. Provincia neotectónica de Cuba.

Aparecen: 1) Unidades neotectónicas (letras azules: $\mathrm{OC}=\mathrm{Occidental}, \mathrm{CO}=\mathrm{Centro-Occidental,} \mathrm{OR}=\mathrm{Oriental}$ ); 2 ) fallas (líneas gruesas negras con letras: $\mathrm{FC}=\mathrm{Cochinos,} \mathrm{FCH}=\mathrm{Cauto}$ límite, $\mathrm{FNC}=$ Nortecubana, $\mathrm{FO}=\mathrm{Oriente}$, $\mathrm{FS}=$ Septentrional, FSC=Surcubana); $\underline{\mathbf{3}}$ ) nudos (círculos y letras rojos: PM=Punta de Maisí (ZAM=Zona de articulación Maisí); 4) sentido de desplazamiento de las placas (flechas gruesas negras: PC=Caribe, $\mathrm{PN}=$ Norteamérica); $\underline{\mathbf{5}}$ ) tensor $\mathrm{O}_{\text {hmáx }}$ (flechas rojas); $\underline{\mathbf{6}}$ ) tipos de corteza (ver leyenda interior: Transicional (1=gruesa, 2=fina), 3=oceánica-modificada); 7) ZC=Zona de cambio estructural (circulo discontinuo rojo). 
La zona de deformación estructural de Cuba, indicada en las Figuras 3 y 5, se ajusta perfectamente con la mayor cantidad de nudos de articulación del plano. En ese entorno se han producido varios eventos sísmicos perceptibles de poca energía, ajustados a una zona de interior de placa.

Tabla 7. Principales terremotos de las Unidades Sismotectónicas Occidental y Centro Occidental.

\begin{tabular}{cccccc}
\hline $\mathrm{N}^{\mathrm{o}}$ & Fecha & $\mathrm{M} / \mathrm{I} / \mathrm{h}(\mathrm{km})$ & Localidad/ Provincia & Epicentro/ Réplicas & Isosistas/ Área $\left(10^{3} \mathrm{~km}^{2}\right)$ \\
\hline 20 & 1880.01 .23 & $6,2 / 8 / 20$ & San Cristóbal/ Pinar del Río & Tierra/ 65 & $\mathrm{SI} / 40$ \\
16 & 1914.02 .28 & $6,2 / 7 / 20$ & Gibara/ Holguín & Mar/ 9 & $\mathrm{~S} / 25$ \\
17 & 1939.08 .15 & $5,6 / 7 / 15$ & Remedios-Caibarién/ Las Villas & Mar/ 24 & $\mathrm{SI} / 19$ \\
21 & 1982.12 .16 & $5,0 / 6 / 20$ & Torriente-Jagüey Grande/ Matanzas & Tierra/ 10 & SI/ \\
19 & 2014.01 .09 & $5,1 / 6 / 10$ & Corralillo/ Las Villas & Mar/ 3 & SI/ \\
23 & 2015.01 .21 & $4,1 / 5 / 16$ & Zapata-Cochinos/ Matanzas & Tierra/ 3 & SI/ \\
\hline
\end{tabular}

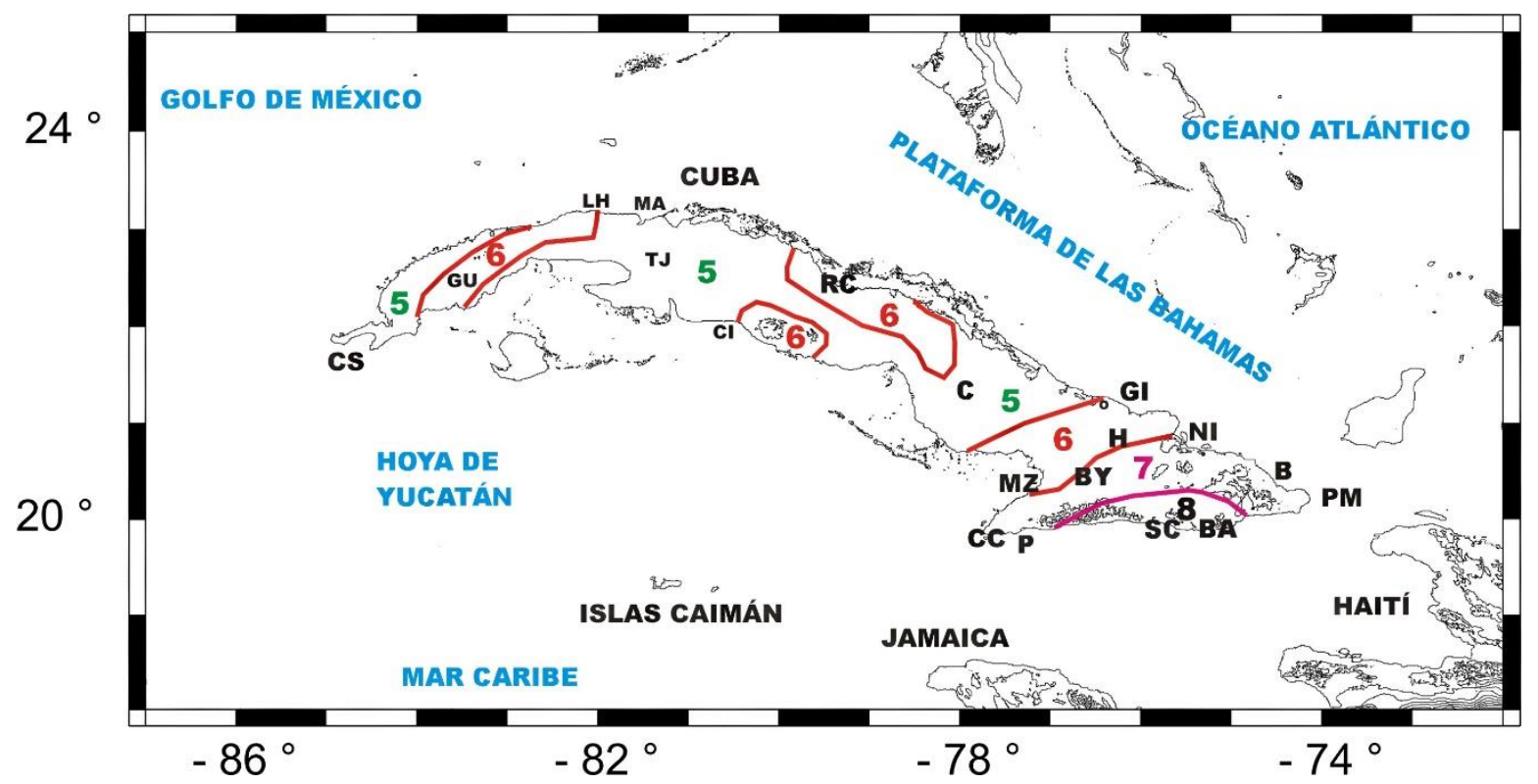

Figura 4. Mapa de Peligrosidad Sísmica de Cuba (Cotilla et al., 1988)

Aparecen: 1) zonas de intensidad sísmica MSK (5-8); 2) localidades: B=Baracoa, BA=Baconao, BY=Bayamo, $\mathrm{C}=$ Camagüey, $\mathrm{CC}=\mathrm{Cabo}$ Cruz; $\mathrm{CI}=$ Cienfuegos, $\mathrm{CS}=$ Cabo de San Antonio, GI=Gibara, GU=Guane, H=Holguín, LH=La Habana, MA=Matanzas, MZ=Manzanillo, NI=Nipe, P=Pilón, PM=Punta de Maisí; RC=RemediosCaibarién, SC=Santiago de Cuba, TJ=Torriente-Jagüey Grande.

Tabla 8. Características principales de la Unidades Sismotectónicas de Cuba.

\begin{tabular}{|c|c|c|c|c|c|c|c|}
\hline$-1-$ & $-2-$ & $-3-$ & $-4-$ & $-5-$ & $-6-$ & $-7-$ & $-8-$ \\
\hline Occidental & Interior de placa & $6,2 / 8 / 25$ & 5 & 100 & 10 & 58 & 5,4 \\
\hline Centro-Occidental & & $6,2 / 8 / 50$ & 10 & & & 53 & 4,5 \\
\hline Oriental & & $6,6 / 8 / 30$ & 15 & & 10 & $<10$ & 0,6 \\
\hline Suroriental & Entre placas & $7,7 / 9 / 60$ & 70 & 80 & 140 & 100 & 0,6 \\
\hline
\end{tabular}


Tabla 9. Comparativa de la actividad sísmica en parte del Caribe Septentrional.

\begin{tabular}{|c|c|c|c|c|c|c|c|}
\hline & $-1-$ & $-2-$ & $-3-$ & $-4-$ & $-5-$ & $-6-$ & $-7-$ \\
\hline Cuba & Norteamérica & $7,7 / 9 / 60$ & $/ 3 / 13$ & 2 & $0,1 / 1,4$ & 80 & 4 \\
\hline Jamaica & Caribe-Gonàve & $7,75 / 9 / 50$ & $/ 4 / 5$ & 8 & $3 / 4,5$ & 60 & 1 \\
\hline La Española & Gonàve-La Española-Puerto Rico & $8,2 / 10 / 90$ & $1 / 18 / 28$ & 13 & $360 / 700$ & 40 & 2 \\
\hline
\end{tabular}

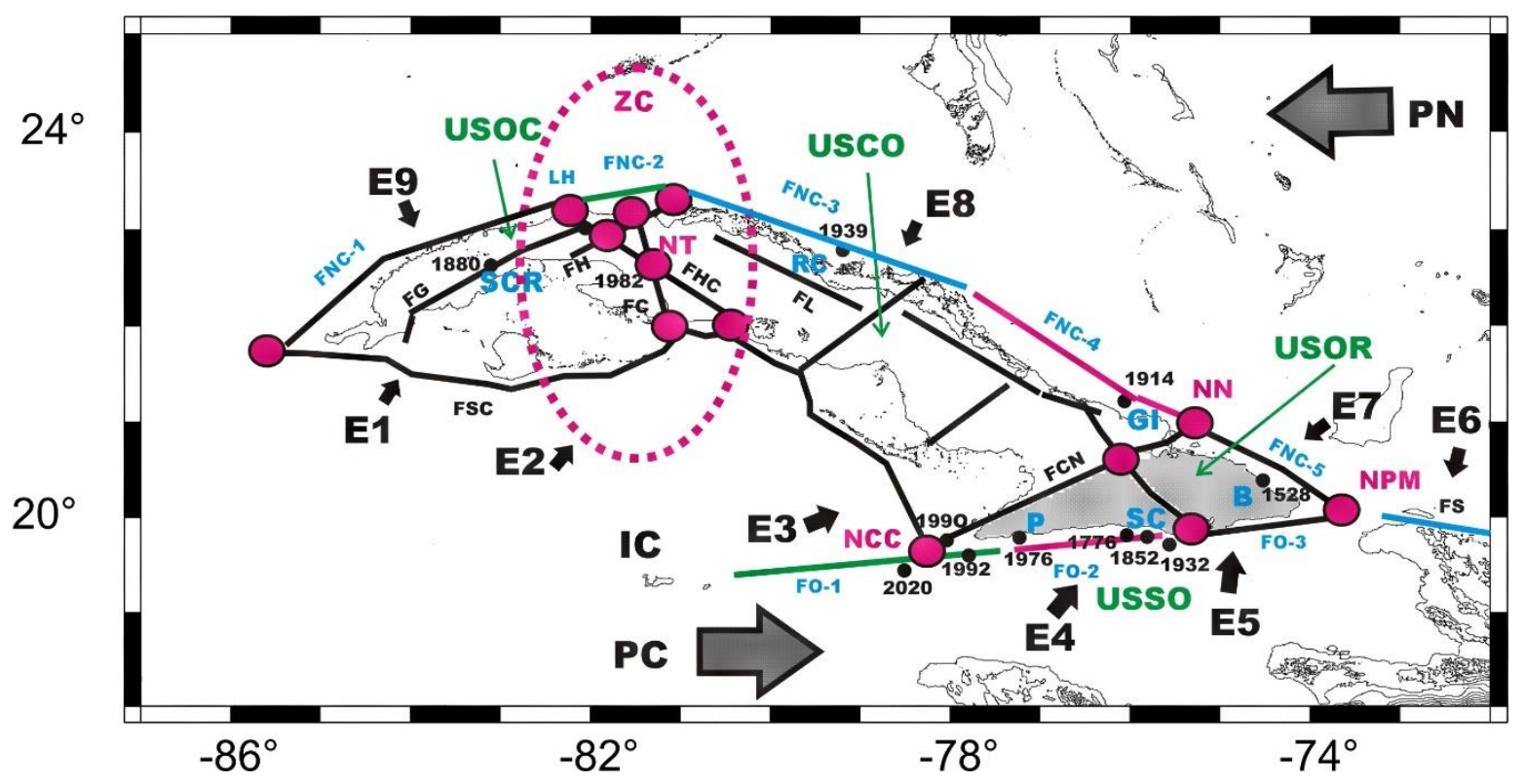

Figura 5. Provincia sismotectónica de Cuba

Aparecen: 1) Unidades sismotectónicas (letras verdes: USOC=Occidental, USCO=Centro-Occidental, USOR=Oriental, USSOR=Suroriental); 2) fallas (líneas negras: $\mathrm{FC}=\mathrm{Cochinos,} \mathrm{FCN}=\mathrm{Cauto}-\mathrm{Nipe}, \mathrm{FG}=\mathrm{Guane}$. $\mathrm{FH}=$ Hicacos, FHC=Habana-Cienfuegos, $\mathrm{FL}=\mathrm{Las}$ Villas, $\mathrm{FNC}=$ Nortecubana (con letras azules, segmentos: FNC-1), $\mathrm{FO}=$ Oriente (con letras azules, segmentos: FO-1), FS=Septentrional, FSC=Surcubana); $\underline{\mathbf{3}}$ ) epicentros (circulo negro con año); 4) tensor $\mathrm{O}_{\text {hmáx }}($ flecha negra gruesa y letra-número E1-5); $\underline{\mathbf{5}}$ ) localidades (letras color azul); $\underline{\mathbf{6}}$ ) nudos (círculos de color con letras en rojo: NCC=Cabo Cruz, NN=Nipe, NPM=Punta de Maisí, NT=Torriente-Jagüey Grande); 7) placas ( $\mathrm{PC}=$ Caribe, $\mathrm{PN}=$ Norteamérica); ) sentido de movimiento de las placas (flechas gruesas de color gris); $\mathrm{ZC}=$ Zona de cambio estructural (circulo discontinuo rojo).

Las fallas sismogénicas de Cuba: 1 ) están segmentadas, y en el caso de nuestro interés: 1.1) Nortecubana (categoría II) tiene 5 segmentos. El segmento de las inmediaciones de Remedios-Caibarién tuvo 2 terremotos con tsunamis en 1931 y 1939 ( $\left.\mathrm{N}^{\circ} 17\right)$; mientras que el segmento aledaño al E hasta Gibara produjo el terremoto de mayor magnitud $(6,2)$ en 1914 ( $\left.\mathrm{N}^{\circ} 16\right)$, pero sin tsunami. Esto implica que sus mecanismos asociados son diferentes. Se aprecia, muy bien, la correspondencia de esta segmentación y las zonas de intensidades sísmicas de la Figura 2; 1.2) Oriente (categoría I) tiene 3 segmentos (Islas Caimán-

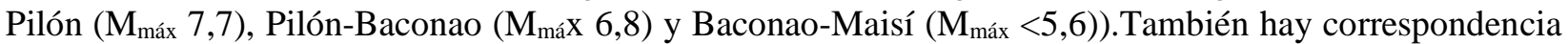
con esta segmentación y la Figura 3; 2) Nortecubana y Oriente no tienen relación de continuidad con la falla Septentrional de La Española. En las inmediaciones de Punta de Maisí, donde el Paso de los Vientos, se configura un nudo de articulación entre las fallas Nortecubana y Oriente, que justifica la dinámica de la 
Unidad Oriental cubana. La falla Septentrional se caracteriza por: 1) tsunamis; 2) $M_{\operatorname{máx}} 8,2$; $\underline{\mathbf{3}}$ ) deformaciones en las terrazas costeras; 4) sismitas. Mientras que los segmentos más orientales de las fallas Nortecubana y Oriente son los de menor magnitud, sin tsunamis locales ni dislocaciones por terremotos.

\section{DISCUSIÓN Y CONCLUSIONES}

Después de todo lo expuesto y discutido anteriormente se puede afirmar que Cuba, como estructura sismogénica del sur de la placa de Norteamérica, tiene: 1) dos tipos de sismicidad; 1.1) entre placas, Caribe-Norteamérica; 1.2) interior de placa, Norteamérica; 2) diferente AS en sus 4 Unidades Sismotectónicas; 3 son de interior de placa y una de entre placas, la más significativa e importante, que está en la Unidad Sismotectónica Suroriental y se distingue por: 2.1) más de 30 terremotos con M $\geq 6,0$; 2.2) $\mathrm{M}_{\text {máx }} 7,7 ; \underline{\mathbf{2 . 3}}$ ) $\sim 100$ fallecidos y $\sim 1.400$ heridos. En esta Unidad está la falla Oriente (categoría I) con 3 segmentos: 1) Islas Caimán-Pilón (M 7,7); 2) Pilón-Baconao (M 6,8); 3 ) Baconao-Maisí (M 5,6). El segundo segmento demuestra la mayor Peligrosidad con la inmensa mayoría de los afectados (muertos, heridos y pérdidas económicas). Ahí está la ciudad de Santiago de Cuba.

Hay otros 2 elementos a considerar en la falla Oriente: 1) no ha generado tsunamis; 2) tiene 2 nudos de articulaciones en la falla Oriente: 2.1) Cabo Cruz (NMGO Figura 2 y NCC Figura 5) donde han ocurrido los terremotos fuertes de $1551\left(\mathrm{~N}^{\circ}\right.$ 9), $1992\left(\mathrm{~N}^{\circ} 5\right), 2007\left(\mathrm{~N}^{\circ} 14\right)$ y $2020\left(\mathrm{~N}^{\circ}\right.$ 2) y que articula la relación entre las fallas Oriente y Cauto Nipe. Ninguno de esos terremotos ha producido víctimas mortales; $\underline{\mathbf{2 . 2}}$ ) Punta de Maisí (NMG1 Figura 2 y NPM Figura 5) que vincula a las fallas Oriente y Nortecubana, en sus segmentos menos activos. Se descartan las "sismo-dislocaciones" y "paleo-sismo-dislocaciones" que algunos especialistas aseguraron existían (Cotilla-Rodriguez, 1993).

La otra estructura sismogénica de importancia es la Nortecubana (categoría II) con 5 segmentos. El tercero de ellos, inmediaciones de Remedios-Caibarién, ha generado tsunamis; pero el cuarto tiene la $\mathbf{M}_{\text {máx }}$ mayor $(6,2)$ en Gibara. Los segmentos más orientales de esta falla y de la de Oriente configuran una zona de articulación en Punta de Maisí, anteriormente mencionada. Estos 2 segmentos tienen las menores magnitudes $(<5,6)$, no producen tsunamis y no tienen relación de continuidad con la falla Septentrional del norte de Haití. Ésta es la más importante de la región (Cotilla et al., 2007).

Finalmente, se presenta la Tabla 10 con un diagnóstico de las posibles afectaciones en 10 ciudades de Cuba por terremotos fuertes. En la primera columna están los datos iniciales y en la segunda las afectaciones más probables. Se aprecia que Santiago de Cuba tiene los mayores niveles. 
Tabla 10. Diagnóstico de afectaciones por terremotos fuertes en Cuba.

\begin{tabular}{ccc} 
& Datos iniciales & Afectaciones \\
\cline { 2 - 3 } Ciudad/ Habitantes & $-1-$ & $-2-$ \\
\hline Baracoa/ 79.797 & Oriental/ 7/ S-S/ 6,0 & PP/ MPO/ 0,3 \\
Bayamo/ 235.107 & Oriental/ 7/ S-N/ 6,6 & N/ MU/ 0,8 \\
Caibarién/ 38.479 & Centro-Occidental/ 6/ S-N/ 5,6 & $\mathrm{S} / \mathrm{PO} / 0,7$ \\
Jagüey Grande/ 87.771 & Centro-Occidental/ 5/ S-N/ 5,5 & $\mathrm{N} / \mathrm{PO} / 0,6$ \\
La Habana/ 2.141.652 & Occidental/ 6/ S-N/ 5,5 & $\mathrm{MP} / \mathrm{PO} / 0,2$ \\
Manzanillo/ 150.999 & Oriental/ 7/ S-N/ 6,6 & $\mathrm{N} / \mathrm{MU} / 0,8$ \\
Mariel/ 45.016 & Occidental/ 5/ S-N/ 5,5 & $\mathrm{P} / \mathrm{PO} / 0,2$ \\
Pilón/ 29.456 & Oriental/ 7/ S-N/ 5,7 & $\mathrm{N} / \mathrm{MU} / 0,5$ \\
San Cristóbal/ 29.119 & Occidental/ 6/ S-N/ 6,2 & $\mathrm{N} / \mathrm{MU} / 1$ \\
Santiago de Cuba/ 444.851 & Oriental/ 8/ S-N/ 6,8 & $\mathrm{N} /$ GP/ 2 \\
\hline Nota: -1-=Región/ Zona sísmica/ Terremoto local-lejano/ M máx; -2- =Tsunami/ Edificaciones/ Personas (\%); \\
GP=Gran parte; MP=Muy poco probable; MPO=Muy pocas; MU=Muchas; N=No; P=Probable; PO=Pocas; \\
PP=Poco probable; S=Sí.
\end{tabular}

AGRADECIMIENTOS: Las Figuras fueron realizadas por Amador García-Sarduy. Los fondos provienen principalmente del Proyecto KUK-AHPAN RTI2018-094827-B-C21.

\section{REFERENCIAS}

Álvarez, L., Cotilla, M. y Chuy, T. Informe final del tema 430.03: Sismicidad de Cuba. Instituto de Geofísica y Astronomía, Academia de Ciencias de Cuba, 1990, 400 pp.

Álvarez, L., Chuy, T., García, J., Moreno, B., Álvarez, H., Blanco, M., Expósito, O., González, O. y Fernández, A.I. An earthquake catalogue of Cuba and neighbouring areas. The Abdus Salam International Centre for Theoretical Physics, Miramare-Trieste, 1999. Internal Report IC/IR/99/1, 60 pp.

Cotilla-Rodríguez, M.O. Una caracterización sismotectónica de Cuba. Tesis de Doctorado, Academia de Ciencias de Cuba, 200 pp., 1993.

Cotilla, M. (2007). Un recorrido por la sismología de Cuba. Editorial Complutense, Universidad Complutense de Madrid, ISBN: 97-8-47-491827.

Cotilla, M. (2010). Cuban seismology. Revista Historia de América, número 143, 43-98.

Cotilla, M. (2011). ¿Tsunamis en Cuba? Revista Física de la Tierra, número 23, 173-197.

Cotilla, M. (2012). Historia sobre la sismología del Caribe Septentrional. Revista Historia de América, $147,111-154$.

Cotilla, M. y Córdoba, D. (2011). Comments about tsunami occurrences in the northern Caribbean. En: Tsunamis, ISBN: 978-953-307-431-3. 
Cotilla, M. y Córdoba, D. (2018). Some glimpses of the tsunamigenic potential of the Caribbean region. En: Google Books, 100 pp.

Cotilla, M. y Udías, A. (1999). Geodinámica del límite Caribe-Norteamérica. Revista de la Sociedad Geológica de España, volumen 12, número 2, 175-186.

Cotilla, M., Córdoba, D. y Calzadilla, M. (2007). Morphotectonic study of Hispaniola. Geotektonika, número 5, 38-62 (en Ruso).

Cotilla, M., Córdoba, D. y Núñez-Cornú, F. (2019). Caracterización sismotectónica de México. Revista Geográfica de América Central, volumen 2, número 63, 103-139.

Cotilla, M., Álvarez, L., Chuy, T. y Portuondo, O. (1988). Peligrosidad sísmica de Cuba (2). Algunos criterios sobre la peligrosidad en zonas de baja actividad del territorio de Cuba. Comunicaciones Científicas Sobre Geofísica y Astronomía, número 5, 15 pp.

González, E., Cotilla, M., Cañete, C., Díaz, J., Carral, R. y Arteaga, F. (2003). Estudio morfoestructural de Cuba. Geografia Fisica Dinamica Quaternaria, número 26, 49-69.

Mann, P. y Burke, K. (1984). Neotectonics of the Caribbean. Review of Geophysics and Space Physics, volume 22, número 4, 309-392.

Rosencratz, E. y Mann, P. (1991). Sea MARC II mapping transform faults in the Cayman trough Caribbean Sea. Geology, número 19, 690-693.

Wiggins-Grandison, M. y Atakan, K. (2005). Seismotectonics of Jamaica. Geophysical Journal International, volumen 160, número 2, 573-580.

\section{SEMBLANZA DE LOS AUTORES}

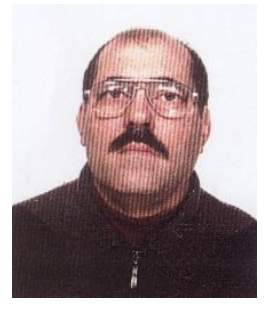

Mario Octavio Cotilla-Rodríguez: Doctor en Ciencias Geográficas [especialidad Sismotectónica, Cuba] y Doctor en Ciencias Físicas [especialidad Geofísica, España]. Profesor Asociado de la Universidad Complutense de Madrid, Facultad de Ciencias Físicas; Cursos de post-grado impartidos 15; Colaboraciones científicas con Instituciones Internacionales (Alemania, España, Francia, México, Noruega, Portugal, República Dominicana, y otras); Participación en Proyectos científicos (>20); Publicaciones científicas en: Revistas (>200), Libros (6), Capítulos de libros (5); Participación en Congresos: Internacionales (>30) y Nacionales (>30)); Revisor (árbitro) de 8 Revistas Científicas; Reconocimientos científicos (4). 